\title{
A MODIFIED GENETIC ALGORITHM FOR FINDING FUZZY SHORTEST PATHS IN UNCERTAIN NETWORKS
}

\author{
A. A. Heidari ${ }^{\text {a }}$, M. R. Delavar ${ }^{\text {b, } *}$ \\ ${ }^{a}$ School of Surveying and Geospatial Eng., College of Eng., University of Tehran, North Kargar Ave., Tehran, Iran \\ ${ }^{\mathrm{b}}$ Center of Excellence in Geomatic Eng. in Disaster Management, School of Surveying and Geospatial Eng., College of Eng., \\ University of Tehran, North Kargar Ave., Tehran, Iran \\ Email: mdelavar@ut.ac.ir
}

Commission IV, WG II/4

KEY WORDS: Genetic Algorithm, Shortest Path Problem, Uncertainty, Quality Assessment, Optimization

\begin{abstract}
:
In realistic network analysis, there are several uncertainties in the measurements and computation of the arcs and vertices. These uncertainties should also be considered in realizing the shortest path problem (SPP) due to the inherent fuzziness in the body of expert's knowledge. In this paper, we investigated the SPP under uncertainty to evaluate our modified genetic strategy. We improved the performance of genetic algorithm (GA) to investigate a class of shortest path problems on networks with vague arc weights. The solutions of the uncertain SPP with considering fuzzy path lengths are examined and compared in detail. As a robust metaheuristic, GA algorithm is modified and evaluated to tackle the fuzzy SPP (FSPP) with uncertain arcs. For this purpose, first, a dynamic operation is implemented to enrich the exploration/exploitation patterns of the conventional procedure and mitigate the premature convergence of GA technique. Then, the modified GA (MGA) strategy is used to resolve the FSPP. The attained results of the proposed strategy are compared to those of GA with regard to the cost, quality of paths and CPU times. Numerical instances are provided to demonstrate the success of the proposed MGA-FSPP strategy in comparison with GA. The simulations affirm that not only the proposed technique can outperform GA, but also the qualities of the paths are effectively improved. The results clarify that the competence of the proposed GA is preferred in view of quality quantities. The results also demonstrate that the proposed method can efficiently be utilized to handle FSPP in uncertain networks.
\end{abstract}

\section{INTRODUCTION}

Finding shortest path in a known network can be regarded as a popular concern among different engineering fields. Shortest path problem (SPP) can be investigated using a directed graph, which is studied using its edges and vertices (Antonio et al., 1992). In this task, the primary purpose is to attain the minimum length path (Effati and Jafarzadeh, 2007). Geoscience experts usually resolve the SPP in transportation planning, robot route planning and traffic tasks (Chuang Kung, 2005). The SPP has been inspected comprehensively. Up to now, various classes of algorithms are developed to tackle this task efficiently such as Dijkstra strategy (Sedeno-Noda and Raith, 2015) and Bellman-Ford (Goldberg and Radzik, 1993) approaches. Besides the traditional approaches, several novel and revised procedures have been established recently. It has been proved that the bi-criterion version of crisp SPP can be treated as a NP-hard problem. Hence, metaheuristic approaches are also useful to tackle SPP, especially for largescale and real-time requests (Mohemmed et al., 2008).

Trevizan and Veloso (2014) introduced depth-based SPP techniques (Trevizan and Veloso, 2014). Pulido et al. verified multi-objective arrangement of SPP using lexicographic preferences (Pulido et al., 2014). Takaoka suggested a notable procedure in data spreading among all pairs SPP (Takaoka, 2014). It is required to propose more competent approaches to understand SPP. With regard to difficulty of SPP; metaheuristics can also determine superior routes in a proper time (Mohemmed et al., 2008). Pervious researches also support the benefits of neural networks (NN) in handling SPP (Ahn et al., 2001). The genetic algorithm (GA) has been applied to realize different aspects of SPP (Ahn and Ramakrishna, 2002). Their paper revealed that GA outperforms NN-based methods in resolving SPP. In 2008, PSO was tested to solve SPP and the achieved results demonstrated that the PSO-based routes are better than those of GA (Mohemmed et al., 2008). In 2010, an ant colony technique (ACO) has been proposed to assess SPP (Ghoseiri and Nadjari, 2008).

In real networks, uncertainty exists in the body of arcs and vertices. With regard to the inherent fuzziness of measurements and expert's knowledge, uncertainties cannot be ignored in realizing the SPP. Although in conventional SPP, the masses of the boundaries are real quantities, most realworld tasks have inaccurate parameters (i.e. costs, measurements, demands, weights, time and capacities). Hence, solving SPP under fuzzy conditions (FSPP) is a motivating problem; subsequently the decision maker will be able to think through risk of the routes. The FSPP is reasonably different from the certain SPP with crisp numbers. In FSPP, fuzzy numbers can be used to model the uncertain aspects of the

\footnotetext{
* Corresponding author
} 
problem. Also, FSPP involves operations on fuzzy numbers that cannot be completed by default methods.

For the first time, Dubois and Prade (1980) suggested FSPP. Their method utilized fuzzy ordering relation and considered modification of the Ford-Moore-Bellman (FMB) technique. Blue et al. (2002) proposed a technique which discovers a cut rate to control the number of investigated routes, and then utilized a revised form of the $k$-shortest path (crisp) strategy. Up to now, several works have been reported about FSPP. Mahdavi et al. (2009) developed a dynamic programming strategy to tackle the fuzzy shortest chain task via an appropriate ranking technique. Tajdin et al. (2010) designed a dynamic programming strategy to handle uncertain SPP in networks having mixed fuzzy values via $\alpha$-cuts. Okada and Soper (2000) presented a model that assigns a possibility to each arc of the shortest path. Lin and Chen (1994) assigned a membership degree to all shortest routes and proposed a fuzzybased technique to explore the best vital edge in route network. Klein (1991) proposed a method that helps decision makers to set a threshold for membership degree and attain promising paths. Ji et al. (2007) proposed different models to tackle SPP in fuzzy situations.

Here, an effective strategy is proposed to obtain the shortest path of a sampler robot in an uncertain network. Performance of genetic algorithm (GA) is improved to investigate a class of SPPs on networks with vague arc weights. As a robust method, the GA is adapted and assessed to challenge the fuzzy SPP (FSPP) with uncertain edges. In the next section, the problem is introduced and then the modified GA and results are presented in detail.

\section{FUZZY SHORTEST PATH PROBLEM (FSPP)}

In order to design the mathematical model of FSPP, a directed acyclic network is considered, Net $=(v, A)$, with a set of nodes, $\quad v=\{1,2, \ldots, n\}$, and a number of arcs, $A$. Each arc is symbolized by $(s, t) \in A$. In this network, only one directed $\operatorname{arc}(s, t)$ from " $s$ " to" $t$ " are allowed. Furthermore, the vertices of an acyclic directed network $\operatorname{Net}=(v, A)$ is reordered so that $s<t$ for every $(s, t) \in A$. Each path can be represented by (Ji et al., 2007):

$x=\left\{x_{s t} \mid(s, t) \in A\right\}, x_{s t} \in\{0,1\}$

where $x_{s t}=1$ shows that the $\operatorname{arc}(s, t)$ is a part of the route. This structure is shown in Figure 1.

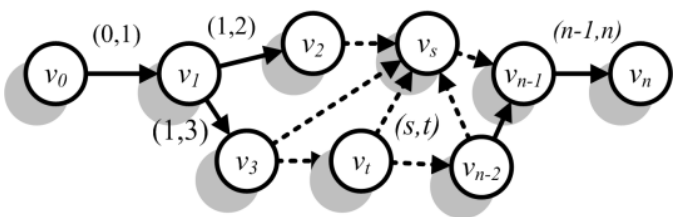

Figure 1. Illustration of a Net

It has been exposed that $x=\left\{x_{s t} \mid(s, t) \in A\right\}$ is considered as a path in a Net if and only if (Ji et al., 2007):

$$
\begin{aligned}
& \sum_{(s, t) \in A} x_{s t}-\sum_{(t, s) \in A} x_{t s}=\left\{\begin{array}{rlrl}
1 & s & =1 \\
0 & 2 \leq s & \leq n-1 \\
-1 & s & =n
\end{array}\right. \\
& x_{s t}=0 \text { or } 1 \quad \forall(s, t) \in A
\end{aligned}
$$

For all $(s, t) \in A$, let the arc lengths $L_{s t}$ be considered as fuzzy variables. $L=\left\{L_{s t} \mid(s, t) \in A\right\}$ is given, then the length of each route $x$ is

$D(x, L)=\sum_{(s, t) \in A} L_{s t} x_{s t}$

In FSPP, the length of each route is fuzzy with respect to the allocated fuzzy values to different arcs. Hence, the main task is to minimize the expected length of pathways. The following model is considered for this purpose (Ji et al., 2007). First the next definition should be provided. For each route $x^{\prime}$, path $x$ is identified as the expected SP (ESP) if

$E[D(x, L)] \leq E\left[D\left(x^{\prime}, L\right)\right]$

where $E[D(x, L)]$ shows the expected shortest route (ESR) length. To discover the ESR, the mathematical model of the FSPP is as:

$\operatorname{minimize} E\left[\sum_{(\mathrm{s}, \mathrm{t}) \in A} m_{\mathrm{st}} x_{\mathrm{st}}\right]$

subject to

$\sum_{(1, t) \in A} x_{1 t}-\sum_{(t, 1) \in A} x_{t 1}=1$,

$\sum_{(s, t) \in A} x_{s t}-\sum_{(t, s) \in A} x_{t s}=0, \quad 2 \leq s \leq n-1$

$\sum_{(n, t) \in A} x_{n t}-\sum_{(t, n) \in A} x_{t n}=-1$,

$x_{s t} \in\{0,1\}, \quad \forall(s, t) \in A$.

where $m$ is an integer value. In the next section, the fuzzy simulation is presented.

\subsection{Fuzzy simulation}

In order to transfigure the uncertain objective and restrictions to their corresponding deterministic variants, fuzzy programming can be utilized. However, this technique is not often effective and easy to implement in some cases. Here, fuzzy simulation is employed to estimate uncertain functions of the model. Before simulation, some definitions should be provided.

The credibility of each fuzzy occurrence can be described as the average of its corresponding possibility and necessity. These concepts are described in Ji et al. (2007). Let $z$ be a fuzzy variable and its membership function $(M F)$ is $\mu(x)$ and $r$ is a real value, then the following equations are true:

$$
\begin{aligned}
& \operatorname{Pos}\{z \geq r\}=\sup _{w \geq r} \mu(w) \\
& \operatorname{Nec}\{z \geq r\}=1-\operatorname{Pos}\{z<r\} \\
& \text { Cred }\{z \geq r\}=0.5 \times(\operatorname{Pos}\{z \geq r\}+\operatorname{Nec}\{z \geq r\})
\end{aligned}
$$

For example, a trapezoidal fuzzy variable, $z$ is described by $\left(z_{1}, z_{2}, z_{3}, z_{4}\right)$ of crisp values with $z_{1}<z_{2}<z_{3}<z_{4}$ and following $M F$, Pos, Nec and Cred:

$\mu(x)=\left\{\begin{array}{cc}\left(x-z_{1}\right) /\left(z_{2}-z_{1}\right) & z_{1} \leq x \leq z_{2} \\ 1 & z_{2} \leq x \leq z_{3} \\ \left(x-z_{4}\right) /\left(z_{3}-z_{4}\right) & z_{3} \leq x \leq z_{4} \\ 0 & \text { otherwise }\end{array}\right.$
$\operatorname{Pos}(z \leq 0)=\left\{\begin{array}{cc}1 & z_{2} \leq 0 \\ z_{1} /\left(z_{1}-z_{2}\right) & z_{1} \leq 0 \leq z_{2} \\ 0 & \text { otherwise }\end{array}\right.$ 


$$
\begin{gathered}
\operatorname{Nec}(z \leq 0)=\left\{\begin{array}{cc}
1 & z_{4} \leq 0 \\
z_{3} /\left(z_{3}-z_{4}\right) & z_{3} \leq 0 \leq z_{4} \\
0 & \text { otherwise }
\end{array}\right. \\
\operatorname{Cred}(z \leq 0)=\left\{\begin{array}{cc}
1 & z_{4} \leq 0 \\
\left(2 z_{3}-z_{4}\right) / 2\left(z_{3}-z_{4}\right) & z_{3} \leq 0 \leq z_{4} \\
0.5 & z_{2} \leq 0 \leq z_{3} \\
z_{1} / 2\left(z_{1}-z_{2}\right) & z_{1} \leq 0 \leq z_{2} \\
0 & \text { otherwise }
\end{array}\right.
\end{gathered}
$$

Another definition is as follows, which is based on Liu and Liu (2002).

$$
E[z]=\int_{0}^{+\infty} \operatorname{Cred}(z \geq r) d r-\int_{-\infty}^{0} \operatorname{Cred}(z \leq r) d r
$$

where $E(z)$ shows the expected value of $z$. For example, the $E(z)$ for trapezoidal numbers is as follows:

$E[z]=0.25 \times \sum_{i=1}^{4} z_{i}$

Based on these concepts, fuzzy simulation can be performed via the following phases:

Step 1: Set $E=0$.

Step 2: randomly create $q_{1 j}, q_{2 j}, q_{3 j}, \ldots, q_{m j}$ based on $\varepsilon$-level sets of $L_{1}, L_{2}, \ldots, L_{m}$ and indicate $q_{j}=\left(q_{1 j}, q_{2 j}, q_{3 j}, \ldots, q_{m j}\right)$, $, j=1,2, \ldots, N$ correspondingly, where $\varepsilon$ is a small amount.

Step 3: Set $z_{1}=D\left(x, q_{1}\right) \wedge D\left(x, q_{2}\right) \wedge \ldots \wedge D\left(x, q_{N}\right)$, $z_{2}=D\left(x, q_{1}\right) \vee D\left(x, q_{2}\right) \vee \ldots \vee D\left(x, q_{N}\right)$.

Step 4: Create random value $r$ based on interval $\left[\mathrm{z}_{1}, \mathrm{z}_{2}\right]$.

Step 5: If $r \geq 0$, then $E \leftarrow E+C r\{D(x, L) \geq r\}$.

Step 6: If $r<0$, then $E \leftarrow E-C r\{D(x, L) \leq r\}$.

Step 7: Repeat 4 to 6 phases for REP times.

Step 8: $E[D(x, L)]=z_{1} \vee 0+z_{2} \wedge 0+E \cdot\left(z_{2}-z_{1}\right) / R E P$.

where $C r($.$) function computes the credibility of its inside part.$ Fuzzy simulation is beneficial to estimate the following uncertain function.

$U: x \rightarrow E[D(x, L)]$

To estimate $U, q_{i k}$ is created using $\varepsilon$-level arrangements of fuzzy values $L_{i}, i=1,2, \ldots, \mathrm{m}$, correspondingly, where $\varepsilon$ is a small value around zero. Set $q_{k}=\left(q_{1 k}, q_{2 k}, q_{3 k}, \ldots, q_{m k}\right)$ and $\mu\left(q_{k}\right)=\mu_{1}\left(q_{1 k}\right) \wedge \mu_{2}\left(q_{2 k}\right) \wedge \ldots \wedge \mu_{m}\left(q_{m k}\right)$. For $\quad r \geq 0 \quad$ and $r<0$, the credibility is calculated by following equations, respectively (Ji et al., 2007):

$$
\begin{aligned}
& C r\left\{D\left(x, q_{j}\right) \geq r\right\}=\frac{1}{2}\left(\begin{array}{l}
\max _{j=1,2, \ldots, N}\left\{\mu\left(q_{k}\right) \mid D\left(x, q_{j}\right) \geq r\right\} \\
+1-\max _{j=1,2, \ldots, N}\left\{\mu\left(q_{k}\right) \mid D\left(x, q_{j}\right)<r\right\}
\end{array}\right) \\
& C r\left\{D\left(x, q_{j}\right) \leq r\right\}=\frac{1}{2}\left(\begin{array}{l}
\max _{j=1,2, \ldots, N}\left\{\mu\left(q_{k}\right) \mid D\left(x, q_{j}\right) \leq r\right\} \\
+1-\max _{j=1,2, \ldots, N}\left\{\mu\left(q_{k}\right) \mid D\left(x, q_{j}\right)>r\right\}
\end{array}\right)
\end{aligned}
$$

where $N$ is a large number.

\section{MODIFIED GENETIC ALGORITHM (MGA)}

GA technique can be regarded as a robust optimization mechanism. This technique inspires the biological experience of genetic evolution (Ahn and Ramakrishna, 2002). The crucial idea is that the genetic pool of a certain population theoretically holds the solution, or a superior solution, to a specified adaptive task (Ahn and Ramakrishna, 2002).
Collaboration of dissimilar chromosomes may lead to better solutions. Throughout reproduction and crossover phases, new genetic mixtures happen and, lastly, a generation can receive a better gene from the parents. Using genetic operators, current population should be evolved in an iterative process.

Ever since the GA technique was designed by Holland (Alvarenga et al., 2007) to realize combinatorial tasks, it has emerged as an efficient meta-heuristic mechanism for tackling several network design problems. In the employed model, as the vertices of the network increase, the final set of routes can be very large. Due to the increasing complexity of this model, the GA is used to find the appropriate set of routes. Every GA algorithm should perform three phases: chromosome structure, crossover step and mutation. The structure of conventional GA is presented in Figure 2.

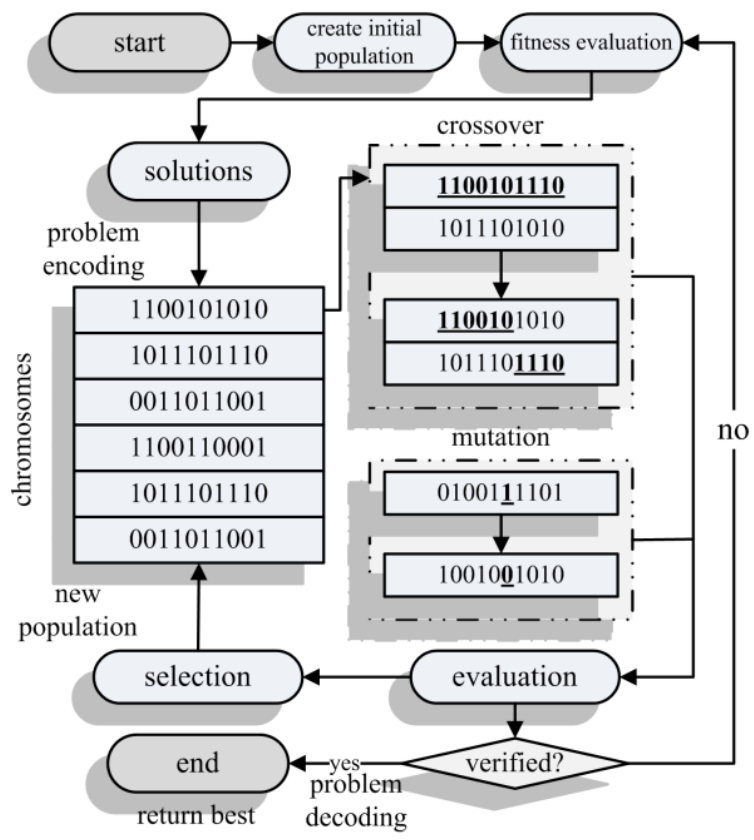

Figure 2. The conventional GA flowchart

For initialization, these steps can be performed:

Step 1: Set $u=0$ and $p_{0}=1$.

Step 2: Select index $m$ randomly like $\left(p_{1}, m\right)$

Step 3: $u \leftarrow u+1$ and $p_{1}=m$.

Step 4: Repeat 2 and 3 phases until $p_{1}=n$.

Step 5: achieve the chromosome $\left(p_{1}, p_{2}, \ldots, p_{u-1}\right)$.

In crossover operation, for two chromosomes, $\left(p_{1}, p_{2}, \ldots, p_{\mathrm{k}}\right)$ and $\left(p_{1}^{\prime}, p_{2}^{\prime}, \ldots, p_{\mathrm{k}^{\prime}}^{\prime}\right)$, if there are shared vertices among them, then one of them can be randomly selected, say $p_{\mathrm{i}}=p_{\mathrm{i}}$. Hence, the following chromosomes are generated:

$\left(p_{1}, p_{2}, \ldots, p_{i}, p_{i^{\prime}+1}^{\prime}, \ldots, p_{k^{\prime}}^{\prime}\right),\left(p_{1}^{\prime}, p_{2}^{\prime}, \ldots, p_{i}^{\prime}, p_{i+1}, \ldots, p_{k}\right)$

For mutation step, first, an integer value should be selected randomly from set $\{1,2,3, \ldots, k\}$, presented by $i$. Then, a route $\left(p_{i^{\prime}+1}^{\prime}, \ldots, p_{k^{\prime}}^{\prime}\right)$ is designed from $\mathrm{p}_{\mathrm{i}}$ to $n$ using a similar procedure of chromosome encoding. Lastly, a new chromosome $\left(p_{1}, p_{2}, \ldots, p_{i}, p_{i^{\prime}+1}^{\prime}, \ldots, p_{k}^{\prime}\right)$ can be generated.

In modified GA-based fuzzy strategy, the rank-based evaluation of genes is determined chaotically. The logistic map is used to generate chaotic values inside interval $(0,1)$. This classic 
chaos-based signal can be observed in nonlinear dynamics of organic populace showing disordered manners (Heidari et al., 2015), and is formulated as

$a_{k+1}=\alpha a_{k}\left(1-a_{k}\right)$

where $a_{k}$ denotes the $k$ th chaos number and $k$ shows the iteration number. Clearly, $a$ is inside $(0,1)$ with opening condition $\mathrm{a}_{0}$ is inside $(0,1)$. In continue, $\alpha=4$ is utilized.

Then, the rank-based evaluation is formed as:

eval $\left(P_{i}\right)=a_{k}\left(1-a_{k}\right)^{i-1}, i=1,2, \ldots$, pop - size

Hence the GA-based fuzzy strategy is implemented using the following steps:

Step 1: Initialize population size chromosomes $P_{k}$, $k=1,2, \ldots$, pop-size at random

Step 2: Compute the objective values of all paths using used fuzzy simulation.

Step 3: Calculate the fitness of every chromosome (gene). The rank-based assessment measure is formulated as

$$
\begin{aligned}
\operatorname{eval}\left(P_{i}\right) & =a_{k}\left(1-a_{k}\right)^{i-1}, \quad i=1,2, \ldots, \text { pop }- \text { size } \\
a_{k+1} & =4 a_{k}\left(1-a_{k}\right), a \in(0,1)
\end{aligned}
$$

Step 4: Select the genes for a new generation.

Step 5: Update the genes using crossover and mutation.

Step 6: Repeat steps 2-5 during certain loops.

Step 7: Return the best path.

\section{EXPERIMENTAL RESULTS}

In order to substantiate the performance of the proposed strategy, a robotic task is investigated. In these scenarios, a mobile robot should explore the shortest route to record some soil samples from different stations in uncertain situation. In these implementations, first, 23 sampling station and 40 possible arcs among them are considered to generate the initial topology of path network. After obtaining required soil material from each station, the robot should move toward the next station to complete the soil sampling task. Due to the various limitations in time and energy, the robot should reach to the last station by following the best expected shortest trajectory. The tested network is depicted in Figure 3. The MF of arc lengths is also exposed on each route.

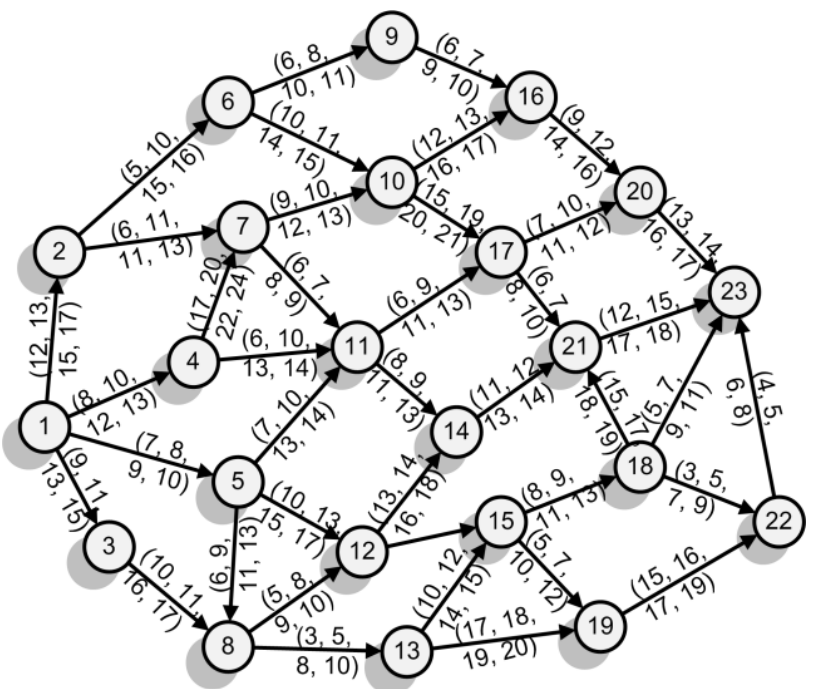

Figure 3. Examined network with 23 nodes (The station IDs are reported on every node, while edge values demonstrate cost of the paths in terms of triangular fuzzy values)
For these experiments, evaluated methods are substantiated using MATLAB R2012a (7.14) on a T6400@4 GHz Intel Core (TM) PC with 4 GB of RAM. After sensitivity study, the GA and MGA methods are evaluated with these parameters: pop_size: 40, total generations: 1000 , total fuzzy simulations: 5000, crossover: 0.4 , mutation: 0.3 .

The optimal route recognized by GA is $1,5,11,17,21,23$ and the length of path is 52.50. The best solution found by MGA is also $1,5,11,17,21,23$, and the length is 52.50 but with better computational performance. The convergence history of the MGA in 10 independent trials with 100 and 200 generations in each test is reported in Table 1. From Table 1, it can be seen

\begin{tabular}{|c|c|c|c|c|}
\hline 竞 & 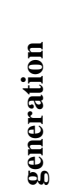 & 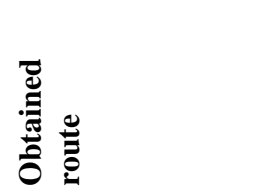 & 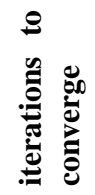 & 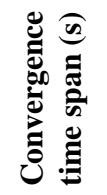 \\
\hline Test-1 & 100 & $1,5,11,17,21,23$ & 17 & 4.117 \\
\hline Test-2 & 100 & $1,5,11,17,21,23$ & 4 & 1.319 \\
\hline Test-3 & 100 & $1,5,11,17,21,23$ & 23 & 5.764 \\
\hline Test-4 & 100 & $1,5,11,17,21,23$ & 19 & 4.801 \\
\hline Test-5 & 100 & $1,5,11,17,21,23$ & 5 & 1.445 \\
\hline Test-6 & 200 & $1,5,11,17,21,23$ & 59 & 14.513 \\
\hline Test-7 & 200 & $1,5,11,17,21,23$ & 12 & 3.112 \\
\hline Test-8 & 200 & $1,5,11,17,21,23$ & 28 & 7.699 \\
\hline Test-9 & 200 & $1,5,11,17,21,23$ & 6 & 1.502 \\
\hline Test-10 & 200 & $1,5,11,17,21,23$ & 33 & 8.633 \\
\hline
\end{tabular}
that MGA can obtain fuzzy shortest length after 4 iterations.

Table 1 . Convergence history of MGA in robotic scenario

In addition, MGA-based FSPP has validated in chorus with GA-based version. The obtained path of MGA-based fuzzy strategy during 33 iterations in test-10 is exposed in Figure 4. The length of $1 \rightarrow 5 \rightarrow 11 \rightarrow 17 \rightarrow 21 \rightarrow 23$ route is 52.50 . However, the MGA-based FSPP algorithm can successfully outperform GA-based FSPP in most of the runs. For example, the computed path of GA-based FSPP after 33 repetitions in test-10 is depicted in Figure 5. It can be detected that the GAFSPP result is $1 \rightarrow 5 \rightarrow 12 \rightarrow 14 \rightarrow 21 \rightarrow 23$, which is not consistent at the same condition.

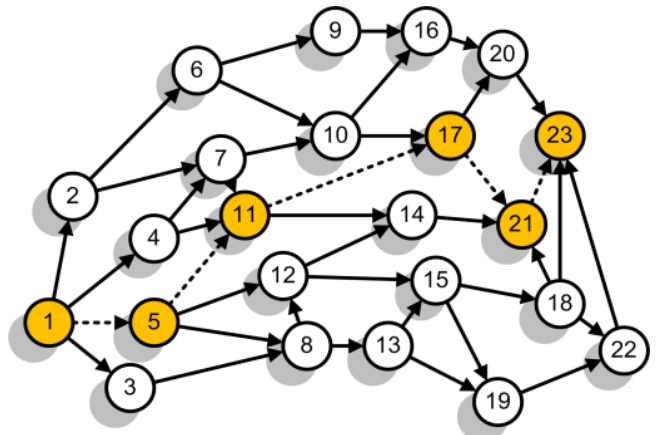

Figure 4. The optimal route of MGA-FSPP in Test-10 


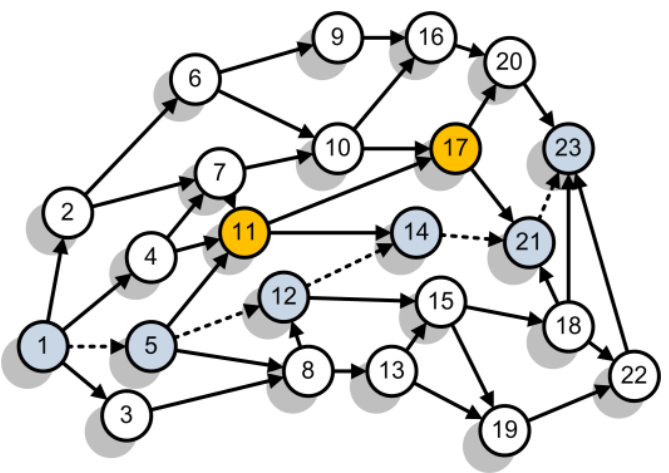

Figure 5. The sub-optimal route of GA-FSPP in Test-10

To investigate the convergence of the MGA, the algorithm is tested with dissimilar parameters. Results reveal that solutions converge effectively and maximum relative error $(R E)$ is $0.936 \%$. The $R E$ value for $Z$ variable can be determined based on:

$R E(Z)=\left(Z_{\text {actual }}-Z_{\text {min }}\right) / Z_{\text {min }} \times 100$

where $Z_{\text {actual }}$ is the actual value of $Z$ and $Z_{\min }$ shows the minimum value of $Z$.

To evaluate the MGA for different networks, it is substantiated on various random topologies with $n$ nodes and $m$ paths. These networks are generated using SPACYC (SPLIB) software ( $\mathrm{Ji}$ et al., 2007). The expected shortest route is investigated using MGA and GA in each random network for 30 times to obtain the biggest $R E$ value. The $R E$ values are reflected in Table 2 . From Table 2, it can be seen that the $R E$ value of MGA is less than $8.0927 \%$. This rate shows that the MGA is also competent for large scale soil sampling robotic networks.

\begin{tabular}{lllll}
\hline Network-ID & $\boldsymbol{n}$ & $\boldsymbol{m}$ & MGA & GA \\
\hline Net-1 & 300 & 1200 & 5.6208 & 7.5594 \\
Net-2 & 400 & 1600 & 3.2719 & 9.8980 \\
Net-3 & 500 & 1500 & 2.3078 & 8.7492 \\
Net-4 & 600 & 2400 & 3.4463 & 13.4362 \\
Net-5 & 700 & 2100 & 8.0927 & 19.2831 \\
Net-6 & 800 & 3200 & 5.0054 & 5.2208 \\
Net-7 & 900 & 2700 & 3.0360 & 3.3130 \\
Net- 8 & 1000 & 3000 & 4.0412 & 4.1233 \\
\hline
\end{tabular}

Table 2. RE Results of GA, MGA on random networks

For bigger networks such as Net-6, Net-7 and Net-8, the difference amongst $R E$ values is reduced. With regard to $R E$ values, the simulations confirm that not only the proposed procedure can outperform GA, but also the qualities of the paths are effectively enriched. These results are also compared in Figure 6.

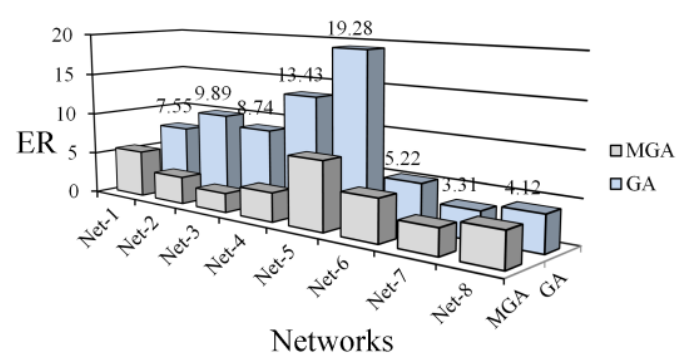

Figure 6. Quality assessment of MGA-FSPP and GA-FSPP based on different random networks
The CPU times of GA and MGA is shown in Table 3. The worst time of MGA is 418.86 (s), while the GA time is not better than 440.34 (s) for larger networks. However, the time of GA and MGA is comparable for some networks such as Net1, Net-2, Net-4 and Net-5. The RE and time results also demonstrate that the premature convergence problem is mitigated effectively. The results also demonstrate that the proposed GA-based technique can competently be used to resolve FSPP in uncertain networks.

\begin{tabular}{lllll}
\hline Network-ID & $\boldsymbol{n}$ & $\boldsymbol{m}$ & MGA (s) & GA (s) \\
\hline Net-1 & 300 & 1200 & 51.91 & 57.28 \\
Net-2 & 400 & 1600 & 64.44 & 63.545 \\
Net-3 & 500 & 1500 & 76.97 & 83.235 \\
Net-4 & 600 & 2400 & 87.71 & 92.185 \\
Net-5 & 700 & 2100 & 148.57 & 142.305 \\
Net-6 & 800 & 3200 & 187.95 & 209.43 \\
Net-7 & 900 & 2700 & 261.34 & 308.775 \\
Net-8 & 1000 & 3000 & 418.86 & 440.34 \\
\hline
\end{tabular}

Table 3. Total running times of GA, MGA on random networks

\section{CONCLUSIONS}

In order to handle SPP task in uncertain networks, a new practical GA-based strategy for obtaining shortest route in fuzzy networks is evaluated. The expected shortest route model is utilized to explore target paths. To validate the performance of proposed approach, a robotic task is studied. In addition, to evaluate the MGA for dissimilar networks, it is substantiated on uncertain networks with random topologies. The results of relative errors and time comparisons show the superiority of the MGA-based FSPP. The evaluation can also be used in other uncertain networks including transportation systems. For future, different fuzzy models can be applied to tackle the SPP.

\section{REFERENCES}

Ahn, C.W., Ramakrishna, R.S., 2002. A genetic algorithm for shortest path routing problem and the sizing of populations. Evolutionary Computation, IEEE Transactions on 6, pp. 566579.

Ahn, C.W., Ramakrishna, R.S., Kang, C.G., Choi, I. C., 2001. Shortest path routing algorithm using hopfield neural network. Electron. Lett. 37 (19), pp. 1176-1178.

Antonio, J.K., Huang, G.M., Tsai, W.K., 1992. A fast distributed shortest path algorithm for a class of hierarchically clustered data networks. IEEE Transactions on Computers 41 (6), pp. 710-724.

Alvarenga, G.B., Mateus, G.R., de Tomi, G., 2007. A twophase genetic and set partitioning approach for the vehicle routing problem with time windows. Computers and Operations Research 34, pp. 1561-1584.

Blue, M., Bush, B., Puckett, J., 2002. Unified approach to fuzzy graph problems. Fuzzy Sets and Systems 125, pp. 355368.

Chuang Kung, J.Y., 2005. The fuzzy shortest path length and the corresponding shortest path in a network, Comput. Oper. Res. 32, pp. 1409-1428. 
Dubois, D., Prade, H., 1980. Fuzzy Sets and Systems: Theory and Applications, Academic Press, New York.

Effati, S., Jafarzadeh, M., 2007. Nonlinear neural networks for solving the shortest path problem. Applied Mathematics and Computation 189 (1), pp. 567-574.

Ghoseiri, K., Nadjari, B., 2010. An ant colony optimization algorithm for the bi-objective shortest path problem. Applied Soft Computing 10 (4), pp. 1237-1246.

Goldberg, A.V., Radzik, T., 1993. A heuristic improvement of the Bellman-Ford algorithm. Applied Mathematics Letters 6 (3), pp. 3-6.

Heidari, A.A., Abbaspour, R.A. and Jordehi, A.R., 2015. An efficient chaotic water cycle algorithm for optimization tasks. Neural Computing and Applications, doi: 10.1007/s00521-0152037-2.

Ji, X., Iwamura, K., Shao, Z., 2007. New models for shortest path problem with fuzzy arc lengths, Applied Mathematical Modelling 31, pp. 259-269.

Klein, C.M., 1991. Fuzzy shortest paths, Fuzzy Set. Syst. 39, pp. 27-41.

Lin, K., Chen, M., 1994. The fuzzy shortest path problem and its most vital arcs, Fuzzy Set. Syst. 58, pp. 343-353.

Liu, B., Liu, Y.K., 2002. Expected value of fuzzy variable and fuzzy expected value model, IEEE Trans. Fuzzy Syst. 10, pp. 445-450.

Mahdavi, I., Nourifar, R., Heidarzade, A., Mahdavi-Amiri, N., 2009. A dynamic programming approach for finding shortest chains in a fuzzy network. Appl. Soft Comput. 9, pp.503-511.

Mohemmed, A.W., Sahoo, N.C., Geok, T. K., 2008. Solving shortest path problem using particle swarm optimization, Applied Soft Computing 8 (4), pp. 1643-1653.

Okada, S., Soper, T., 2000. A shortest path problem on a network with fuzzy arc lengths, Fuzzy Set. Syst. 109 (1), pp. 129-140.

Pulido, F., L. Mandow, L., de la Cruz, J.L.P., 2014. Multiobjective shortest path problems with lexicographic goalbased preferences. European Journal of Operational Research 239 (1), pp. 89-101.

Sedeno-Noda, A., Raith, A., 2015. A Dijkstra-like method computing all extreme supported non-dominated solutions of the biobjective shortest path problem, Comput. Oper. Res. 57, pp. 83-94.

Tajdin, A., Mahdavia, I., Mahdavi-Amiri, N., SadeghpourGildeh, B., 2010. Computing a fuzzy shortest path in a network with mixed fuzzy arc lengths using $\alpha$-cuts. Comput. Math. Appl. 60, pp. 989-1002.

Takaoka, T., 2014. Sharing information for the all pairs SPP. Theoret Comput Sci 520 (6), pp. 43-50.

Trevizan, F.W., Veloso, M.M., 2014. Depth-based shortsighted stochastic shortest path problems. Artificial Intelligence 216, pp. 179-205. 